\title{
MANEJO DEL PÓLIPO ENDOCERVICAL EN LA POSMENOPAUSIA
}

\author{
Félix Dasio Ayala Peralta ${ }^{1,2, a}$, Antonio Luna Figueroa ${ }^{1,2, b}$, César Carranza Asmat ${ }^{1,2, b}$, \\ Enrique Guevara Ríos ${ }^{1,2, b}$, Janet Evelyn Castilla Galvan ${ }^{2, c}$, Yeraldine De la Cruz Leiva ${ }^{1, d}$, \\ Dresly Aguilar Goicochea ${ }^{1, d}$, Aldo Apcho Cruzado ${ }^{1, d}$
}

\begin{abstract}
RESUMEN
Los pólipos endocervicales son las patologías benignas más comunes del cuello uterino, cuya etiología aún es desconocida. Aparecen en el 2 al $5 \%$ de las mujeres. En general se originan en canal endocervical y en menos frecuencia desde el ectocérvix. Se presenta el caso de una mujer de 51 años de edad cuya fecha de última menstruación fue hace 2 años y acude a consultorio de climaterio a un control de rutina, asintomática y al examen ginecológico se visualiza una tumoración polipoidea de $1 \times 0,5 \mathrm{~cm}$ de color rosado con pedículo sésil acortado, a quien se le realiza polipectomía y cuyo estudio histológico revela pólipo endocervical sin signos de malignidad.
\end{abstract}

Palabras clave: Pólipo endocervical; Polipectomía; Posmenopausia (Fuente: DeCS BIREME).

\section{MANAGEMENT OF ENDOCERVICAL POLIPES IN POSMENOPAUSE}

\begin{abstract}
Endocervical polyps are the most common benign pathologies of the cervix, whose etiology is still unknown. They appear in 2 to $5 \%$ of women. In general they originate in the endocervical canal and less frequently from the ectocervix. The case of a 51-year-old woman whose last menstruation date was 2 years ago is presented and goes to a climacteric office for routine, asymptomatic control and a $1 \times 0,5 \mathrm{~cm}$ color polypoid tumor is visualized in the gynecological examination. Pink with shortened sessile pedicle, to whom polypectomy is performed and whose histological study reveals endocervical polyp without signs of malignancy.
\end{abstract}

Keywords: Endocervical polyp; Polypectomy; Postmenopause (Source: MeSH NLM).

\section{INTRODUCCIÓN}

Los pólipos endocervicales (PEC) se originan en el canal endocervical, que generalmente son lesiones benignas, que aparecen en el 2 al $5 \%$ de las mujeres ${ }^{1-3}$ y más de la mitad de ellas se encuentran entre los 40 y 65 años de edad, de este grupo $45 \%$ son posmenopáusicas ${ }^{4}$, pero pueden malignizarse en 0,2 a $1,5 \%$ de los casos $^{5}$.

Estos pólipos se caracterizan por estar constituidos de epitelio cilíndrico, razón por la cual son conocidos como pólipos cervicales verdaderos. Macroscópicamente protruyen a través del orificio cervical externo en forma de un pedículo vascular, su coloración varía de rojo cereza a violeta, textura suave y flexible. Pueden manifestarse en forma simple o múltiple, siendo la primera la presentación más frecuente ${ }^{6}$.
Mayormente los pólipos miden menos de $1 \mathrm{~cm}$ de diámetro, pero en ocasiones presentan mayor diámetro, denominándolos pólipos gigantes, no obstante, no existe un consenso en el punto de corte pues algunos autores los llaman así cuando miden más de $2 \mathrm{~cm}$ y otros cuando son más de $4 \mathrm{~cm}^{3,7-9}$.

Frecuentemente son asintomáticos, siendo hallados mediante un examen ginecológico de rutina, mientras que los pólipos sintomáticos se pueden manifestar con sangrado intermenstrual, poscoital y posmenopáusico ${ }^{6}$.

La histogénesis de los pólipos cervicales es desconocida; pero, los factores causales incluyen a inflamación crónica, estímulo estrogénico ${ }^{3,10}$, cuerpos extraños ${ }^{10,11}$ y la multiparidad $^{10}$.

Universidad Nacional Mayor de San Marcos. Departamento Académico de Ginecología y Obstetricia. Lima- Perú.

2 Instituto Nacional Materno Perinatal. Lima-Perú

a Médico Gíneco Obstetra.Profesor Ordinario de la Facultad de Medicina de la Universidad Nacional Mayor de San Marcos. Lima- Perú. Código ORCID https://orcid.org/0000-0002-2830-3789

b Médico GínecoObstetra. Profesor de la Facultad de Medicina de la Universidad Nacional Mayor de San Marcos. Lima-Perú.

Médico Anatomopatólogo.

Alumnos del 6to año de medicina. Curso de Ginecología y Obstetricia. Sede Instituto Nacional Materno Perinatal. Lima-Perú.

Citar como: Ayala FD, Luna A, Carranza C, Guevara E, Castilla JE, De La Cruz Y, Aguilar D, Apcho A. Manejo del pólipo endocervical en la posmenopausia.

Rev Peru Investig Matern Perinat 2019; 8(3): 45-8

DOI https://doi.org/10.33421/inmp.2019165

Recibido: 08-08-2019 Aceptado: 27-09-2019 
El manejo en mujeres asintomáticas puedes ser expectante ${ }^{12-14} ; \quad y$ en casos seleccionados que ocasionan sangrado o flujo se deben extirparse mediante polipectomía, polipectomía bajo visualización colposcópica, polipectomía con legrado endocervical, polipectomía con histeroscopía y polipectomía con legrado endocervical y endometrial|15-17. En la mayoría de los casos la escisión se puede llevar a cabo en el consultorio mediante prensión de la base con pinzas Pfoester o de anillo y torsión del pólipo (polipectomía). La polipectomía no requiere anestesia. El sangrado después de la extirpación es raro y puede controlarse con tapón con gasa o cauterio químico. Es recomedable remitir la pieza quirúrgica para estudio anatomopatológico.

\section{DESCRIPCIÓN DE CASO CLÍNICO}

Se presenta el caso de una mujer de 51 años de edad con historia ginecoobstétrica de menarquia a los 14 años, régimen catamenial 3/28, fórmula obstétrica G1P1001, fecha de último parto hace 17 años, última menstruación hace dos años, sin pareja sexual desde hace 17 años. Sin antecedentes personales de importancia.

La paciente acude a consultorio externo de climaterio por presentar síntomas vasomotores y cefalea de leve intensidad; asimismo refiere incremento de peso corporal. Niega sangrado vaginal. Al examen físico; Presión arterial: $120 / 90 \mathrm{mmHg}$, frecuencia cardiaca: 78/minuto, talla: $1,51 \mathrm{~m}$, peso: $70,3 \mathrm{~kg}$, IMC: $30,8 \mathrm{Kg} / \mathrm{m}^{2}$, circunferencia abdominal: $105 \mathrm{~cm}$. Al examen ginecológico mediante especuloscopía se evidencia trofismo vaginal disminuido, cérvix con orificio cervical externo bicomisural por donde protruye una tumoración polipoidea de $1 \times 0,5 \mathrm{~cm}$ de color rosado con pedículo sésil acortado; además hay escaso flujo vaginal de aspecto blanquecino y no se evidencia sangrado vaginal (Figura 1). Al tacto vaginal; vagina reseca, acortada, cérvix a $1 \mathrm{~cm}$ del fondo de saco posterior, se palpa tumoración polipoidea pediculada

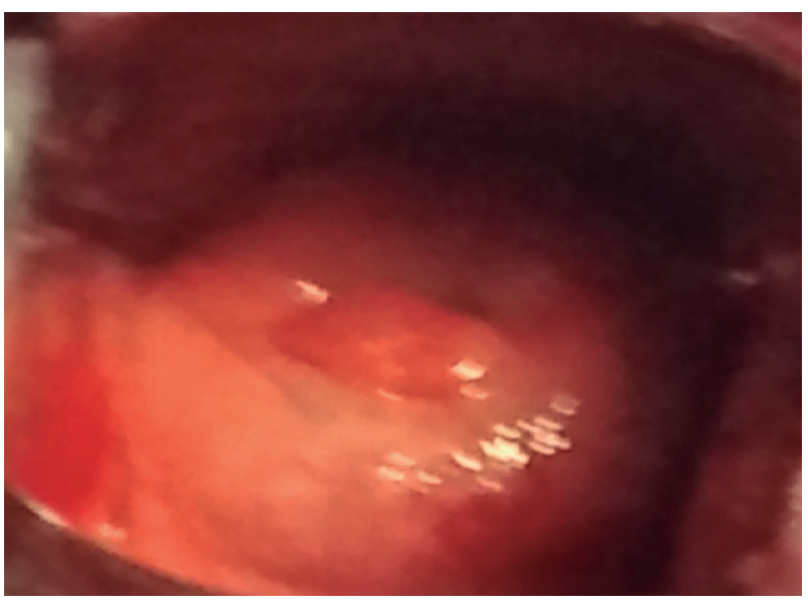

Figura 1. Tumoración polipoidea de $1 \times 0,5 \mathrm{~cm}$ de color rosado con pedículo sésil acortado que protruye por OCE. de aproximadamente $1 \times 0,5 \mathrm{~cm}$ que protruye por canal endocervical, útero anteversoflexo cuyas dimensiones son $6 \times 4 \times 3 \mathrm{~cm}$, no doloroso a la movilización, anexos libres. Resto del examen físico sin alteraciones.

Se le realizaron exámenes de laboratorio de rutina como hemograma, glucosa basal cuyos valores fueron normales; sin embargo, el perfil lipídico se encontró alterado: Colesterol total: $259 \mathrm{mg} / \mathrm{dL}$, HDL-COL: $49 \mathrm{mg} / \mathrm{dL}$, LDLCOL : >130 mg/dL. Triglicéridos:223mg/dL. Urocultivo negativo. Se tomó la muestra cervical para Papanicolaou cuyos hallazgos del informe citológico cervical revelan como negativo para lesión intraepitelial, inflamación moderada, hematíes (+), metaplasia escamosa y atrofia. En estudio de ecografía transvaginal se evidencia útero anteverso de $66 \times 37 \times 45 \mathrm{~mm}$ con endometrio lineal de $2,5 \mathrm{~mm}$, mioma intramural tipo 3 y ovarios involutivos.

El procedimiento quirúrgico en la consulta ambulatoria, consistió previa asepsia de genitales externos, canal vaginal y cervical con yodopovidona, se realizó polipectomía mediante clampaje del pólipo con pinza Foester y torsión en rotación de $360^{\circ}$ para extirpar el pólipo; luego la muestra se envía a patología. El resultado anátomo patológico reporta formación polipoide de $1 \times 0,5 \times 0,3 \mathrm{~cm}$ blanco grisáceo; cuyo diagnóstico histopatológico revela pólipo endocervical (Figuras 2a y2b).

Los diagnósticos finales del caso fueron pólipo endocervical, postmenopausia natural, obesidad, síndrome genitourinario de la menopausia, dislipidemia e hipertensión arterial. Se le realiza control postpolipectomía y no se halla complicaciones propias de la intervención quirúrgica, no obstante, se evidencia flujo vaginal blanquecino. Se le indica Estriol 0,1\% crema vaginal, clindamicina en óvulos vaginales, atorvastatina $20 \mathrm{mg}$ por 30 días, complementándolo con dieta saludable e incremento de ejercicios. La evolución clínica fue favorable (figura 3).

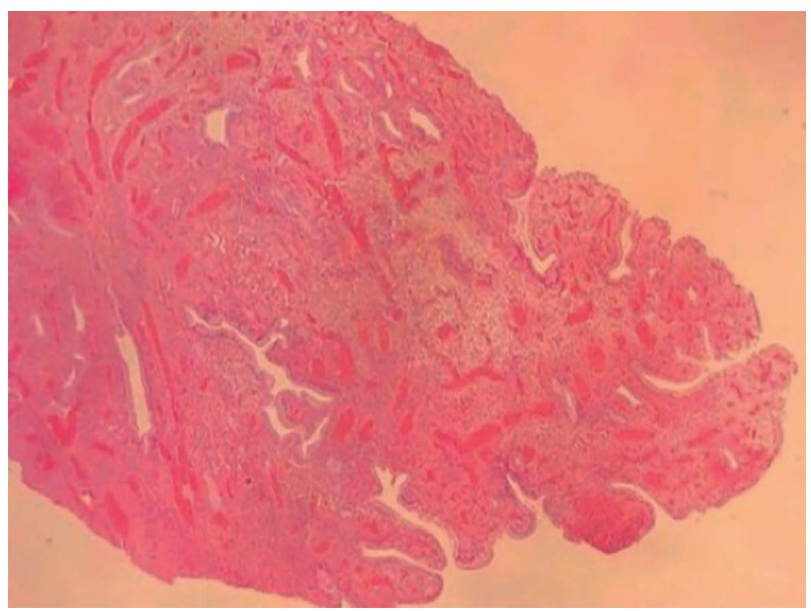

Figura 2a. Histopatología de pólipo endocervical: Epitelio mucinoso columnar alto, tipo endocervical cubre la superficie y las criptas 


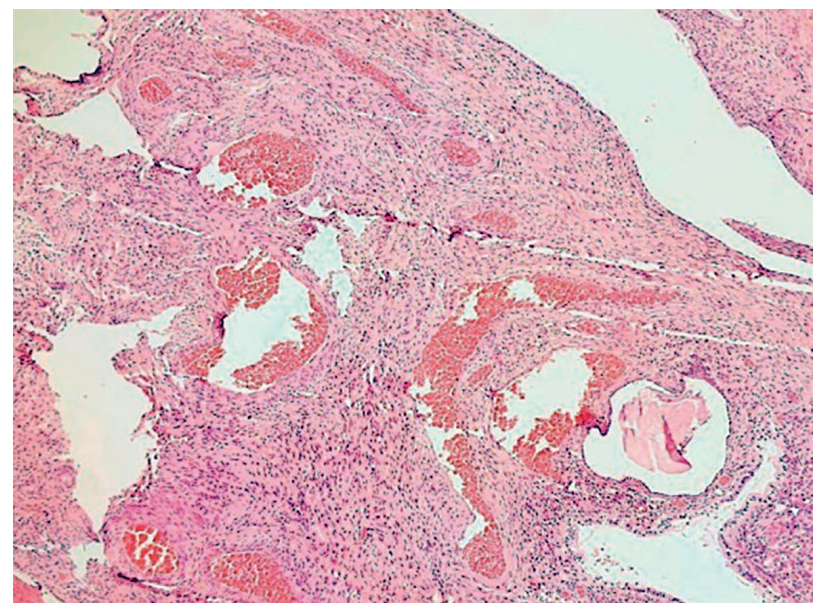

Figura 2b. Histopatología de pólipo endocervical: Vasos sanguíneos centrales dilatados y congestivos, rodeados por glándulas endocervicales e infiltrado inflamatorio crónico.

\section{DISCUSIÓN}

Los pólipos endocervicales son producto de la hiperplasia epitelial glandular, suelen ser benignos en su mayoría, pero pueden malignizar en un $0,1-1,5 \%$ de casos; así mismo, son el segundo tipo de pólipo más diagnosticado en la consulta ginecológica ${ }^{1}$.

De acuerdo a su localización pueden clasificarse en 2 subtipos: endocervicales cuando se encuentren dentro de la cavidad cervical y exocervicales cuando estén presentes en la superficie externa del cuello uterino ${ }^{1,6}$. Los pólipos endocervicales son considerados los verdaderos tipos de pólipos cervicales ya que están constituidos por epitelio cilíndrico y desarrollan un pedículo vascular que protruye por el OCE ${ }^{5}$. Los pólipos endocervicales son el subtipo más frecuente y están presentes principalmente en mujeres en edad reproductiva y premenopaúsicas ${ }^{1,5}$. En su presentación pueden ser únicos o múltiples en raras ocasiones; suelen ser asintomáticos, pero en algunos casos dan sintomatología como sangrado intermenstrual, postcoital o posmenopaúsico ${ }^{5}$. Se reporta el caso de un pólipo endocervical en una mujer posmenopaúsica y de hallazgo incidental ya que la paciente no reportó sintomatología durante la consulta.

El manejo de pólipo endocervical consiste desde polipectomía en consulta ambulatoria hasta la histerectomía total cuando el pólipo tiene características malignas. Estas opciones deben considerarse teniendo en cuenta entre varios factores la edad de la paciente y el deseo de tener descendencia. Después de la cirugía es necesario realizar un seguimiento cuidadoso ya que los pólipos podrían reaparecer, incluso hay estudios que sugieren que los pólipos podrían preceder al desarrollo de hiperplasia endometrial o adenocarcinoma endometrial ${ }^{18,19}$. Por este motivo es fundamental realizar el estudio histopatológico de todas las muestras extirpadas.

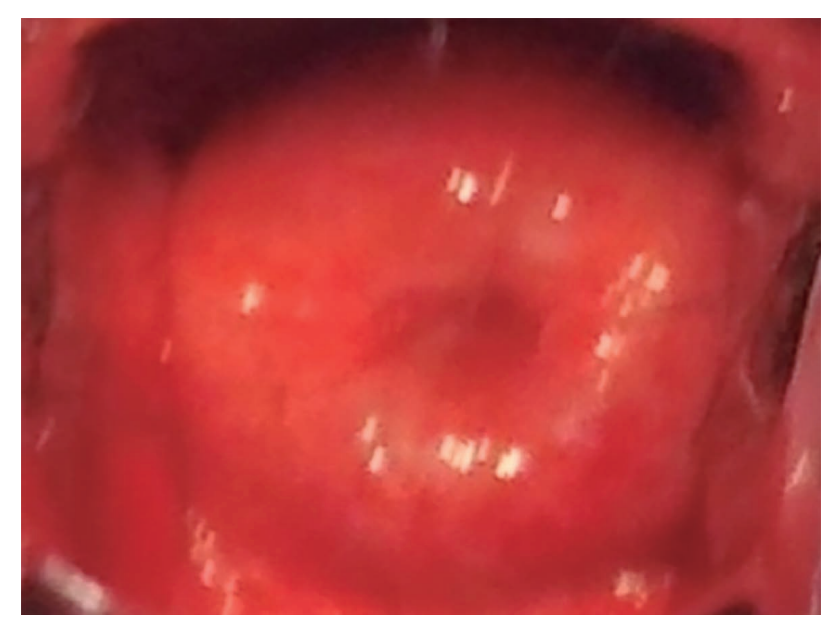

Figura 3. Evolución cervical posterior a la polipectomía.

Los pólipos endocervicales generalmente se consideran lesiones hiperplásicas y muestran cantidades variables de estructuras glandulares y estromales. Por lo general, al estudio histológico se evidencia un estroma edematoso suelto con estructuras vasculares dilatadas. Aunque son poco frecuentes, pueden encontrarse también heterotopías en el cuello uterino, como la presencia de tejido sebáceo en el ectocérvix, la melanosis cervical, el tejido neuroglial en el estroma cervical, cartílago heterólogo en el orificio cervical interno ${ }^{20}$.

\section{Financiamiento: Autofinanciado.}

Conflicto de interés: Los autores declaran no tener algún conflicto de intereses.

\section{REFERENCIAS BIBLIOGRÁFICAS}

1. Tanos V, Berry KE, Seikkula J, Abi E, Stavroulis A, Sleiman Z, Campo R, Gordts S. The management of polyps in female reproductive organs. International Journal of Surgery. 2017;43:7-16. https://doi.org/10.1016/j.ijsu.2017.05.012

2. Ushadevi Gopalan1, Sathiyakala Rajendiran, Ranganathan Karnaboopathy. Clinicopathological analysis of cervical polyps. International Journal of Reproduction, Contraception, Obstetrics and Gynecology Gopalan U et al. Int J Reprod Contracept Obstet Gynecol. 2017;6(4):15261529. DOI: http://dx.doi.org/10.18203/2320-1770. ijrcog20171421

3. Soyer T, Demirdağ G, Güçer S, Orhan D, Karnak I. Giant cervical polyp with mespnephric duct remnants: unusual cause of vaginal bleeding in an adolescent girl. Fetal Pediatr Pathol. 2014; 33(3): 176- 181

4. Younis MT, Iram S, Anwar B, Ewies AA. Women with asymptomatic cervical polyps may not need to see a gyneacologist or have them removed: an observational retrospective study of 1126 cases. Eur J Obstet Gynecol Reprod Biol. 2010; 150(2): 190-194.

5. Levy RA, Kumarapeli A, Spencer HJ, Quick CM. Cervical polyps: Is histologic evaluation necessary? Pathol Res 
Pract. 2016;2012(9):800-803. https://doi.org/10.1016/j. prp.2016.06.010

6. Palacios-Pereira LT, Cervantes-Ramirez L, Espinal-Rodríguez $\mathrm{J}$, Espinal-Madrid J, Sabillón-Vallejo J, Urquia-Osorio H. Pólipo cervical gigante de larga evolución. Reporte de un caso. Archivos de medicina. 2015; 11(4):5. doi: 10.3823/1267

7. Yadav BS, Nandedkar SS, Malukani K, Agrawal P. Multiple giant cervical polyps: A case report with literature review. Indian Journal of Basic and Applied Medical Research 2014; 3(3): 338-343.

8. Anduaga-Aguirre MA, Gonzalez-Mohino MB, Ibero JL. Pólipo endocervical. Medicina general 2002; 45: 524-525.

9. Yi KW, Song SH, Kim KA, Jung WY, Lee JK, Hur JY. Giant endocervical polyp mimicking cervical malignancy: primary excision and hysteroscopic resection. J Minim Invasive Gynecol. 2009; 16(4): 498-500.

10. Grigore M. Giant cervical polyps: Three cases with differents pathologies. J Obstet Gynaecol. 2015; 35(5): 529-530.

11. Abdul MA, Koledade AK, Madugu N. Giant cervical polyp complicating uterine fibroid and masquerading as cervical malignancy. Arch Int Surg. 2012; 2: 39-41.

12. Goldshmid O, Schejter E, Kugler D, Menczer J. Is removal of asymptomatic cervical polyps necessary?: histologic findings in asymptomatic Israeli Jewish women. J Low Genit Tract Dis.2011;15(4):259-262.

13. MacKenzie IZ, Naish C, Rees CM, Manek S. Why remove all cervical polyps and examine them histologically? BJOG.2009;116(8):1127-1129.

14. Younis MT, Iram S, Anwar B, Ewies AA. Women with asymptomatic cervical polyps may not need to see a gynaecologist or have them removed: an observational retrospective study of 1126 cases. Eur J Obstet Gynecol Reprod Biol.2010;150(2):190-194.

15. Long ME, Dwarica DS, Kastner TM et al. Comparison of dysplastic and benign endocervical polyps. J Low Genit Tract Dis.2013;17(2):142-146.

16. Heatley MK. Squamous intraepithelial lesions arising in benign endocervical polyps: a report of 9 cases with correlation to the pap smears, HPV analysis, and immunoprofile. Int J Gynecol Pathol. 2009;28(6):567.

17. Tokunaka M, Hasegawa J, Oba T et al. Decidual polyps are associated with preterm delivery in cases of attempted uterine cervical polypectomy during the first and second trimester. J Matern Fetal Neonatal Med.2014;30:1-3.

18. Inoue $\mathrm{K}$, Tsubamoto $\mathrm{H}$, Hori $\mathrm{M}$, Ogasawara $\mathrm{T}$, Takemura T. A case of endometrioid adenocarcinoma developing 8 years after conservative management for atypical polypoid adenomyoma. Gynecol Oncol Case Rep. 2014;8:21-23.

19. Takahashi H, Yoshida T, Matsumoto T, et al. Frequent b-catenin gene mutations in atypical polypoid adenomyoma of the uterus. Hum Pathol. 2014;45:33-40.

20. Nucci MR, Young RH. Arias-Stella reaction of the endocervix: a report of 18 cases with emphasis on its varied histology and differential diagnosis. Am J Surg Pathol 2004;28: 608-12.

Correspondencia: Félix Dasio Ayala Peralta

Dirección: Jr. Maracaibo $N^{\circ}$ 2153. San Martín de Porres. Lima, Perú

Correo: fayala1401@hotmail.com.

Teléfono: 999227657 